Results: There were a total of 5909 childhood cancer cases; 2959 aged 0-14 years (1659 males, 1300 females) and 2950 aged 1524 years (1590 males, 1358 females). For 0-14-year-old boys, there was statistically significant sinusoidal variation in month of birth for acute non-lymphocytic leukaemia ( $p=0.04$; peak in September) and astrocytoma $(p=0.03$; peak in October). Based on month of diagnosis, there was statistically significant sinusoidal variation in girls for all lymphomas ( $p=0.05$; peak in March) and Hodgkin lymphoma ( $p=0.005$; peak in January), and in boys for osteosarcoma ( $p=0.05$; peak in October). For 15-24-year-olds, there was significant heterogeneity for germ cell tumours $(p=0.04)$, cervical $(p=0.03)$ and female breast carcinoma $(p=0.05)$, based on month of birth, and PNETs $(p=0.05)$ and skin carcinoma $(p=0.05)$, based on month of diagnosis. Significant sinusoidal variation in month of birth for malignant melanoma in females $(p=0.03$; peak in March) and cervical carcinoma ( $p=0.03$; peak in October) was observed.

Conclusions: These findings suggest that seasonal environmental factors around the time of birth or time of diagnosis may be involved in the aetiology of specific diagnostic groups. Further research is needed to study possible aetiological mechanisms and factors. Putative agents include sunlight, pesticides, diet and infections.

\section{THE EPIDEMIOLOGY OF BONE CANCER DIAGNOSED IN 0-49- YEAR-OLDS IN NORTHERN ENGLAND, 1981-2002}

${ }^{1} \mathrm{RG}$ Feltbower, ${ }^{2} \mathrm{R}$ Eyre, ${ }^{2} \mathrm{PW}$ James, ${ }^{2} \mathrm{~K}$ Blakey, ${ }^{2} \mathrm{E}$ Mubwandarikwa, ${ }^{3,4} \mathrm{D}$ Forman, ${ }^{1}$ PA McKinney, ${ }^{2} \mathrm{MS}$ Pearce, ${ }^{2} \mathrm{RJO}$ McNally. ${ }^{1}$ Paediatric Epidemiology Group, Centre for Epidemiology and Biostatistics, University of Leeds, Leeds, UK; ${ }^{2}$ Institute of Health and Society, Newcastle University, Sir James Spence Institute, Royal Victoria Infirmary, Newcastle upon Tyne, UK; ${ }^{3}$ Northern and Yorkshire Cancer Registry and Information Service, Bexley Wing, St James's University Hospital, Leeds, UK; ${ }^{4}$ Cancer Epidemiology Group, Centre for Epidemiology and Biostatistics, University of Leeds, Leeds, UK

\section{doi:10.1136/jech.2009.096701v}

Aims: We aimed to describe incidence and survival patterns for bone cancers diagnosed during 1981-2002 in northern England among 0-39-year-olds.

Methods: 509 cases with a malignant bone tumour were included the analysis and classified using the International Classification of Diseases for Oncology, second edition. Incidence trends were analysed using Poisson regression. Survival rates were calculated using Kaplan-Meier estimation and differences in survival between diagnostic groups assessed using log-rank tests. Cox regression analysis was used to model the probability of survival in relation to age, gender and year of diagnosis.

Results: Overall incidence (per million person years) rates were 3.0 (95\% CI 2.6 to 3.4) for osteosarcoma, 2.1 (1.8 to 2.5) for Ewing sarcoma and 0.8 (0.6 to 1.0) for chondrosarcoma. Incidence of osteosarcoma increased significantly by an average annual rate of $2.6 \%(p=0.02)$ although there was no change in incidence for Ewing sarcoma or chondrosarcoma. Survival improved for Ewing sarcoma (hazard ratio (HR) per annum $0.97,95 \%$ CI 0.94 to 1.00), although patients aged $15-39$ years $(n=93)$ had worse overall survival than those aged $0-14(n=73)$ (HR 1.46, 95\% CI 0.98 to 2.17). There was no improvement in osteosarcoma survival (HR per annum $0.98,95 \%$ CI 0.95 to 1.01 ).

Conclusions: Our data suggested that incidence of osteosarcoma increased significantly in contrast to other bone tumours among 0 39-year-olds and previous findings from the UK and USA. Poorer survival in older Ewing sarcoma patients is consistent with previous studies and may be due to treatment, delays in diagnosis, metastatic disease, site and the stage of the tumour. The failure to improve survival for osteosarcoma patients needs further investigation.

\section{INCIDENCE OF CENTRAL NERVOUS SYSTEM TUMOURS WITH THE USE OF HORMONE REPLACEMENT THERAPY}

V Benson, K Pirie, J Green, G Reeves, V Beral, on behalf of the Million Women Study Collaborators Cancer Epidemiology Unit, University of Oxford, Oxford, UK

doi:10.1136/jech.2009.096701w

Background: Approximately 4300 people are diagnosed with a brain or other central nervous system (CNS) tumour annually in the UK. They have poor prognosis, yet little is known about risk factors of the tumours.

Objectives: To investigate the role of hormone replacement therapy (HRT) and the risk of brain and CNS tumours, specifically gliomas, meningiomas, and acoustic neuromas in post-menopausal women.

Design: Prospective cohort study.

Participants and Setting: 1.1 million post-menopausal women were recruited from breast screening clinics from 1996-2001 in England and Scotland and followed for incident tumours through NHS cancer registration. All CNS tumours and each of the tumour types (glioma, meningioma, and acoustic neuroma) were separate end points in a Cox proportional hazards model for investigation of various measures of HRT use.

Main Outcome Measure: Relative risk (RR) of incident CNS tumours (classified as malignant or benign) relating to the use of HRT, adjusting for age, socioeconomic status, region of residence, height, and body mass index.

Preliminary Results: A total of 1163 post-menopausal women with HRT information were diagnosed with a tumour of the brain or CNS; 517 tumours were classified as glioma, 280 as meningioma, and 113 as acoustic neuroma. Preliminary findings show that current users of HRT were more likely to develop a CNS tumour when compared to never users (RR $1.18,95 \%$ CI 1.03 to 1.35). Findings by type of HRT use and by the histological subtype of the tumours will be presented.

Conclusion: Current users of HRT are at a slightly increased risk of developing CNS tumours.

\section{INTERNATIONAL TRENDS IN PROSTATE CANCER INCIDENCE AND MORTALITY IN 20 COUNTRIES FROM 1980 TO 2002}

${ }^{1} \mathrm{AE}$ Carsin, ${ }^{2} \mathrm{E}$ Masuyer, 'L Sharp, ${ }^{2} \mathrm{P}$ Autier. ${ }^{1}$ National Cancer Registry, Cork, Ireland; 'International Agency for Research on Cancer, Lyon, France

doi:10.1136/jech.2009.096701x

Objective: Worldwide, since the 1980s, there have been major changes in prostate cancer detection and treatment with the availability of the prostate specific antigen (PSA) test and the introduction of hormone therapy and radical prostatectomy. To explore the effect of these advances on the burden of disease, we investigated time trends in prostate cancer incidence and mortality in 20 industrialised countries.

Data Source: Data on prostate cancer incidence during 1980 to 2002 was extracted from volumes 6 to 9 of Cancer Incidence in Five Continents. Mortality data from 1990 to 2002 were obtained from the WHO mortality database.

Methods: Age-standardised rates (ASR), based on the male population aged $\geqslant 50$, were calculated for each year in each country using the European Standard Population. Annual percentage change (APC) in incidence and mortality rates, and the points in time when trends changed, were estimated by fitting join point regression models using Join point software (3.1). Overall percentage change $(\mathrm{OPC})$ during the study period was defined as $\mathrm{OPC}=(1+\mathrm{APC})^{\text {number }}$ of years -1 . Trends for men aged $50-74$ and $\geqslant 75$ years were also analysed. 
Results: In terms of incidence, in 1980, the overall ASR varied widely across countries, ranging from 18 per 100000 in Belarus to 309 per 100000 in Switzerland. Between 1980 and 2002, prostate cancer incidence increased in all 20 countries. The OPC was the smallest in Denmark (+40\%) and highest in Italy (+336\%). Generally, countries with lower incidence in early years had the highest OPCs. In North-America and Australia incidence peaked around 1994, whereas in most European countries rates rose throughout the study period. The increase was most pronounced among men aged 50-74, and in a few countries, the OPC for men aged $\geqslant 75$ years was less than zero. Mortality rates decreased in North-America and some western European countries (eg France, England, Italy, Switzerland), remained stable in others (eg Scotland, Sweden, Denmark) and increased in Eastern Europe. In countries where rates fell, the decline was more pronounced among younger, than older, men. In some countries (eg France), mortality began to fall before incidence peaked.

Conclusions: International variations in prostate cancer incidence and mortality were observed. The different trend in incidence by age suggests an impact of earlier diagnosis/PSA testing. While the decreases in mortality observed in some countries might be a result of improvements in treatment or earlier detection, they could also be affected by changes in death certificate coding or competing causes of death.

\section{Thursday, 10 September}

\section{Parallel session $B$}

\section{Lifecourse obesity}

\section{BODY MASS INDEX THROUGH LIFE AND ADULT MORTALITY: RESULTS FROM THE BRITISH 1946 BIRTH COHORT}

${ }^{1,2}$ BH Strand, 'D Kuh, 'I Shah, ${ }^{2} \mathrm{~J}$ Guralnik, ${ }^{1} \mathrm{R}$ Hardy. ${ }^{1}$ MRC National Survey of Health and Development, MRC Unit for Lifelong Health and Ageing, 33 Bedford Place, London, UK; ' 2 Laboratory of Epidemiology, Demography, and Biometry National Institute on Aging, National Institutes of Health, Bethesda, MD, USA

\section{doi:10.1136/jech.2009.096701y}

Background: Adult body mass index (BMI) has been consistently related to mortality, but little is known about the impact of earlier life BMI on adult mortality.

Objective: Investigate the impact of childhood, adolescent and early adult BMI on premature adult mortality and assess whether any associations are explained by adult BMI.

Design: Cohort study with prospective information on BMI at ages 2, 4, 6, 7, 11, 15, 20, 26 and 36 years, and mortality follow-up from age 26 to 60 years. BMI was standardised at each age, separately for men and women.

Setting: England, Scotland, Wales.

Participants: 2325 males and 2136 females.

Outcome measure: All-cause mortality (332 deaths).

Results: Splines were used to model the non-linear associations between BMI and mortality. In both genders, adult BMI from 20 years onwards showed a consistent U-shaped relationship with adult mortality (overall p-value $<0.05$ for BMI at ages 20, 26 and 36 years). In females, a similar relationship was observed for adolescent BMI at 15 years $(p=0.02)$; the hazard ratio (HR) comparing females with low BMI (2 standard deviations (SDs) below mean) vs mean BMI was 2.96 (95\% CI 1.26 to 6.97). The corresponding HR for females with BMI 2 SDs above the mean was 1.97 (0.95 to 4.10). In males, increased mortality rates were only seen for low adolescent BMI. BMI in childhood was generally not associated with adult mortality. The exception was BMI at age 4 years in females, where a U-shaped relationship was observed ( $p=0.02)$; HR for low BMI (2 SDs below mean) at 4 years vs mean BMI was 2.13 (0.97 to 4.70). The HR for females with BMI 2 SDs above the mean was 1.67 (0.85 to 3.28), and for females with BMI 3 SDs above mean it was 3.08 (1.21 to 7.83). This association was not explained by subsequent BMI change, adult BMI, smoking, childhood social class or adult educational level.

Conclusions: High and low childhood and adolescent BMI are related to adult premature mortality, especially in women. Interventions to reduce under- and overweight in childhood are required to prevent increasing premature adult mortality in more recent cohorts with greater numbers of overweight children.

\section{TRAJECTORIES OF BODY MASS INDEX AND OVERWEIGHT IN EARLY ADULTHOOD AND BLOOD PRESSURE AT 53 YEARS}

A Wills, S Black, G Mishra, D Kuh, R Hardy. MRC Unit for Lifelong Health and Aging, University College London, London, UK

\section{doi:10.1136/jech.2009.096701z}

Background: Body mass index (BMI) is positively associated with blood pressure (BP) throughout adulthood. However, it is not known whether being overweight $\left(\mathrm{BMI}>25 \mathrm{~kg} \cdot \mathrm{m}^{2}\right)$ earlier in adult life is associated with higher BP independent of current BMI, or whether there are sensitive periods for weight gain in adulthood. Objectives: To explore the pathways by which being overweight in adulthood is associated with BP at 53 years.

Design: Prospective birth cohort study with information on BMI at 20, 26, 36, 43 and 53 years and BP at 53 years

Setting: England, Scotland, Wales.

Participants: 1451 males and 1479 females.

Outcome: BP at age 53 years. Censored regression models, accounting for individuals on antihypertensive medication, were used. To investigate a cumulative influence, we estimated the effect of time from being first overweight. To investigate sensitive period(s), we used standardised conditional BMI velocities for each interval. Lastly, an overweight trajectory was defined for each individual based on their status at 26, 36 and 53 years to try to formally assess the evidence for an accumulation or sensitive period pathway.

Results: Overweight at all ages was associated with a higher mean $\mathrm{BP}$ at 53 years. After adjusting for current BMI, only overweight at age 43 in men contained any additional information on BP at 53 years. Men who became overweight at 26 had a SBP $8.7 \mathrm{~mm} \mathrm{Hg}$ higher (95\% CI 4.4 to 13.0) than those first overweight at 53. Similar patterns but smaller associations were seen in women ( $p$ (interaction) $<0.001)$. All periods of adult weight gain $(26-36,36-$ $43,43-53$ years) were associated with a higher BP. The association varied little between periods, in males it ranged from a 2.7$3.6 \mathrm{~mm} \mathrm{Hg}$ increase in SBP per SD increase in BMI velocity. BMI tracked strongly through adulthood, the BMI at 53 years in men first overweight at 26 was $30.9 \mathrm{~kg} \cdot \mathrm{m}^{2}$ (95\% CI 30.5 to 31.4$)$ compared to $26.4 \mathrm{~kg} \cdot \mathrm{m}^{2}$ (95\% CI 26.3 to 26.6) in those first overweight at 53 . Few individuals moved to a normal weight once overweight, this prevented a reliable estimation of the excess risk associated with prolonged overweight and meant it was difficult to disentangle a sensitive period or accumulation pathway using the different trajectories of overweight.

Conclusion: Early adult overweight and all periods of adult weight gain irrespective of earlier BMI were associated with a higher later life BP. Associations at younger ages appear to be largely mediated through BMI tracking. This highlights the importance for later health of preventing overweight in early adulthood. 